\title{
Immunohistochemical expression of CD56 in dog (Canis
}

\section{familiaris) odontogenesis}

Sulette $\mathrm{Nel}^{a}, *$, Marlene van Heerden ${ }^{a}$, Willie van Heerden ${ }^{a}$

${ }^{a}$ Department of Oral Pathology and Oral Biology, School of Dentistry, Faculty of Health

Sciences, University of Pretoria, Oral and Dental Hospital, Bophelo Road, Pretoria

Running Title: Immunohistochemical expression of CD56 in dog odontogenesis

Keywords: CD56; odontogenesis

*Corresponding author: Sulette Nel

PO Box 1266, Pretoria, 0001, South Africa

Tel.: +27123192664

Fax: +27 123212225

Cell: 0827724511

E-mail address: sulette.nel@up.ac.za 
ABSTRACT

Aim: To investigate the expression of CD56 in dog odontogenesis in order to elucidate the expression found in ameloblastomas.

Materials and methods: Immunohistochemical analysis of CD56 expression of developing dog teeth in the bud, cap and bell stages including the remnants of the dental lamina.

Results: Weak CD56 expression was observed in the dental epithelium during the bud stage with intense staining of certain peripheral epithelial cells. Positive staining of epithelial cells was also observed in the cap stage with intense staining of the inner enamel epithelium at this stage. During the bell stage the staining was concentrated on the cervical loop areas. The dental papilla revealed positive staining throughout the cap and bell stages while the dental follicle stained intensely positive throughout all the phases examined. The dental lamina and Serres rests also stained positive for CD56.

Conclusions: The expression of CD56 in dog odontogenic tissue varies according to the stage of tooth development. There is a positive correlation between the positive staining observed in ameloblastomas and their odontogenic cells of origin. 


\section{Introduction}

CD56 (neural cell adhesion molecule, NCAM) is a transmembrane molecule that belong to the immunoglobulin superfamily. ${ }^{1}$ It is a specific marker for natural killer (NK) cells and is also expressed on the surface of various cells including glia and neurones. ${ }^{2,3}$ This molecule mediates homophilic adhesion in certain cell types. ${ }^{1}$ Together with other substrate adhesion molecules, CD56 is associated with processes of emigration and early migration of neural crest cells. CD56 expression is retained after neural crest cells have left the neuroectoderm on their way to the pharyngeal arch destinations and labels a subpopulation of neural crest cells in long tailed monkeys (Macaca fascicularis). ${ }^{4}$ Physiologically, CD56 functions in the cohesion of epithelia, conditioning of cell migration and transmigration via biological membranes and provide means of homing cells in a new environment. ${ }^{5}$ CD56positive subpopulations of human craniofacial muscle-derived cells have been shown to contain lineage committed myogenic cells as well as multipotent cells that are able to form fat and bone tissue. ${ }^{6}$

CD56 is expressed in a variety of tumours including multiple myeloma, ${ }^{7}$ serous neoplasm of the ovary, ${ }^{8}$ neuroblastomas ${ }^{5}$ and neuroendocrine tumours. ${ }^{9}$ The expression thereof is also well documented in ameloblastomas. ${ }^{10,11}$ The origin of these odontogenic tumours seems to be the dental lamina and more specifically the remnants thereof referred to as Serres rests. ${ }^{12}$ It is therefore crucial to examine the tissue of origin to elucidate the staining patterns observed in the associated tumours. To the best of our knowledge, there are currently four studies where CD56 expression was studied in developing mouse teeth ${ }^{13-}$ ${ }^{16}$ while another two studies evaluated the CD56 expression in human impacted third molars. ${ }^{17,18}$ In the latter two cases the tooth crown was already formed and CD56 
expression was only investigated in the ectomesenchymal cells of the dental follicle and third molar pad. ${ }^{17,18}$

It was stated in 2010 that there is still a lack of understanding of the context in which CD56 is expressed in odontogenic epithelium and therefor further investigation is warranted. ${ }^{10}$ In this study we therefor aimed to elucidate the expression of CD56 expression in tooth development in order to understand and explain the staining patterns observed in ameloblastomas.

Although most of the studies on tooth development are done on mice and rats one should be careful in extrapolating results as mice do not have canine or premolar teeth. ${ }^{19}$ Dogs on the other hand have three incisors, one canine, four premolars and two molars in each maxillary quadrant. The same pattern occurs in the mandibular quadrants with the exception that dogs have a third molar in the mandible. ${ }^{19}$ This corresponds with the two incisors, one canine, two premolars and two or three molars in the human dentition. ${ }^{19}$

\section{Materials and Methods}

\section{$2.1 \quad$ Tissue acquisition and processing}

Twenty four foetuses of large breed dogs were obtained under ethical clearance of the Animal Use and Care Committee of the Faculty of Veterinary Sciences, University of Pretoria, South Africa, from female dogs scheduled for elective pregnancy termination. The foetuses were fixed in $10 \%$ buffered formalin and the heads carefully cut into coronal sections, dehydrated and embedded in paraffin wax blocks. Those samples that contained calcified bone or dental hard tissues were decalcified in routine decalcifying solution $(70 \mathrm{ml}$ 
$\mathrm{HNO}_{3}, 50 \mathrm{ml} \mathrm{HCL}, 880 \mathrm{ml}$ distilled water) for 60 minutes and then rinsed in running tap water for 60 minutes before processing. Tissue specimens were sectioned at $3 \mu \mathrm{m}$, stained with haematoxylin and eosin (H\&E), and microscopically examined to select slides with wellformed enamel organs in which the respective odontogenic structures were morphologically clearly identifiable.

Although tooth development occurs as a continuous process, we divided the process into the bud, cap and bell stages in order to differentiate between variations in the staining pattern. The following criteria were used to determine the relevant stage of tooth development. The bud stage was defined as the stage where epithelial cells proliferated into the underlying ectomesenchyme without formation of a cap shape or presence of stellate reticulum (SR). A tooth germ was regarded as being in the cap stage when the enamel organ resembled a cap on a ball of ectomesenchyme and SR cells were observed without the presence of differentiated ameloblasts or odontoblasts. The bell stage was characterized by the advanced features where differentiated ameloblasts and odontoblasts were visible with or without the presence of hard tissue formation. The cervical loop area was defined as the area where the inner enamel epithelium (IEE) and outer enamel epithelium (OEE) meet. The dental lamina breaks up during the bell stage and therefor Serres rests were examined on these slides.

\subsection{Immunohistochemical staining}

Immunohistochemical staining was performed on $3 \mu \mathrm{m}$ sections cut from formalin fixed, paraffin embedded tissue blocks using antibodies to CD56 (clone 123C3, 1:50 dilution, 
DAKO, Carpinteria, CA). Staining was performed on the Ventana Benchmark GX automated system (Ventana Medical Systems, Arizona USA). The protocol of 80 minutes antigen retrieval in CC1 (Ventana), two hours of titrated antibody incubation and detection with the Optiview DAB IHC kit (Ventana) was followed. Controls were included for positive and negative staining. The sections were dehydrated, cleared, mounted with a permanent mounting media and evaluated with a light microscope. Brown cytoplasmic and/or membrane staining was interpreted as positive for the presence of CD56.

\section{Results}

CD56 staining patterns are summarized in table 1. During the bud stage, the odontogenic epithelial cells in general revealed weak positive staining for CD56 with intense positivity in a large portion of the palisaded peripheral cells (Fig.1). Strong and diffuse positivity was also observed in the condensed ectomesenchyme surrounding it. Throughout the cap stage the OEE and SR stained positively for CD56 while intense positivity was observed in the peripheral aspects of the IEE (Fig.2). The central aspect of the IEE, associated with the enamel knot, revealed only weak staining. All through this stage weak staining was observed in the dental papilla while the dental follicle stained intensely positive for CD56. For the period of the bell stage, CD56 staining of the enamel organ seemed to become concentrated at the cervical loops revealing intense positivity of the IEE, stratum intermedium (SI), SR and OEE in this area (Fig.3). Differentiated IEE cells/ameloblasts located more towards the coronal aspect were devoid of any staining. For the duration of this stage the dental pulp and follicle maintained its CD56 expression. On the sections 


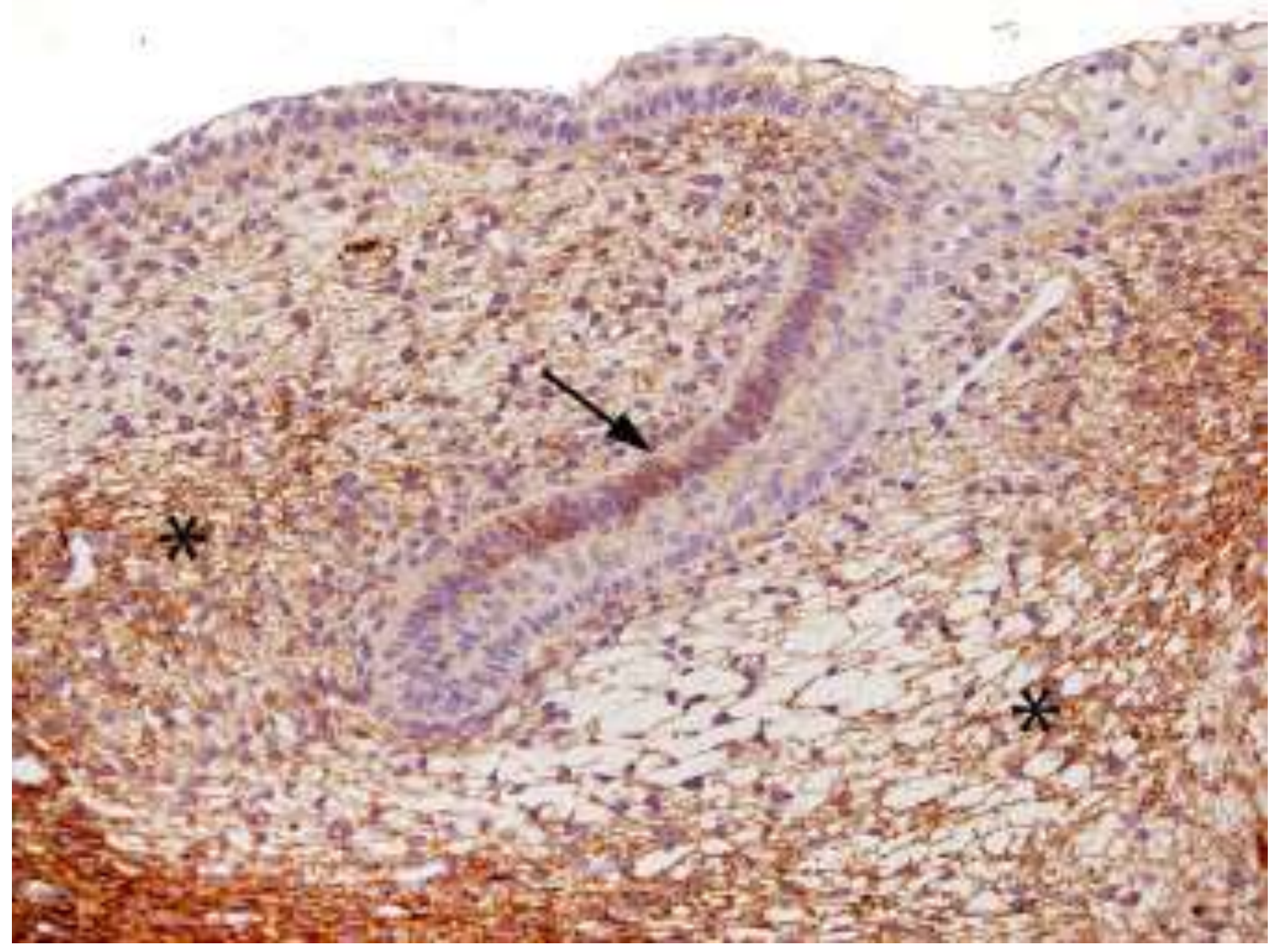

Fig. 1 - Intense CD56 staining of the majority of the palisaded peripheral odontogenic epithelial cells (arrow) in the bud stage with diffuse positivity of the associated condensed ectomesenchyme (asterisks) (original magnification X100). 


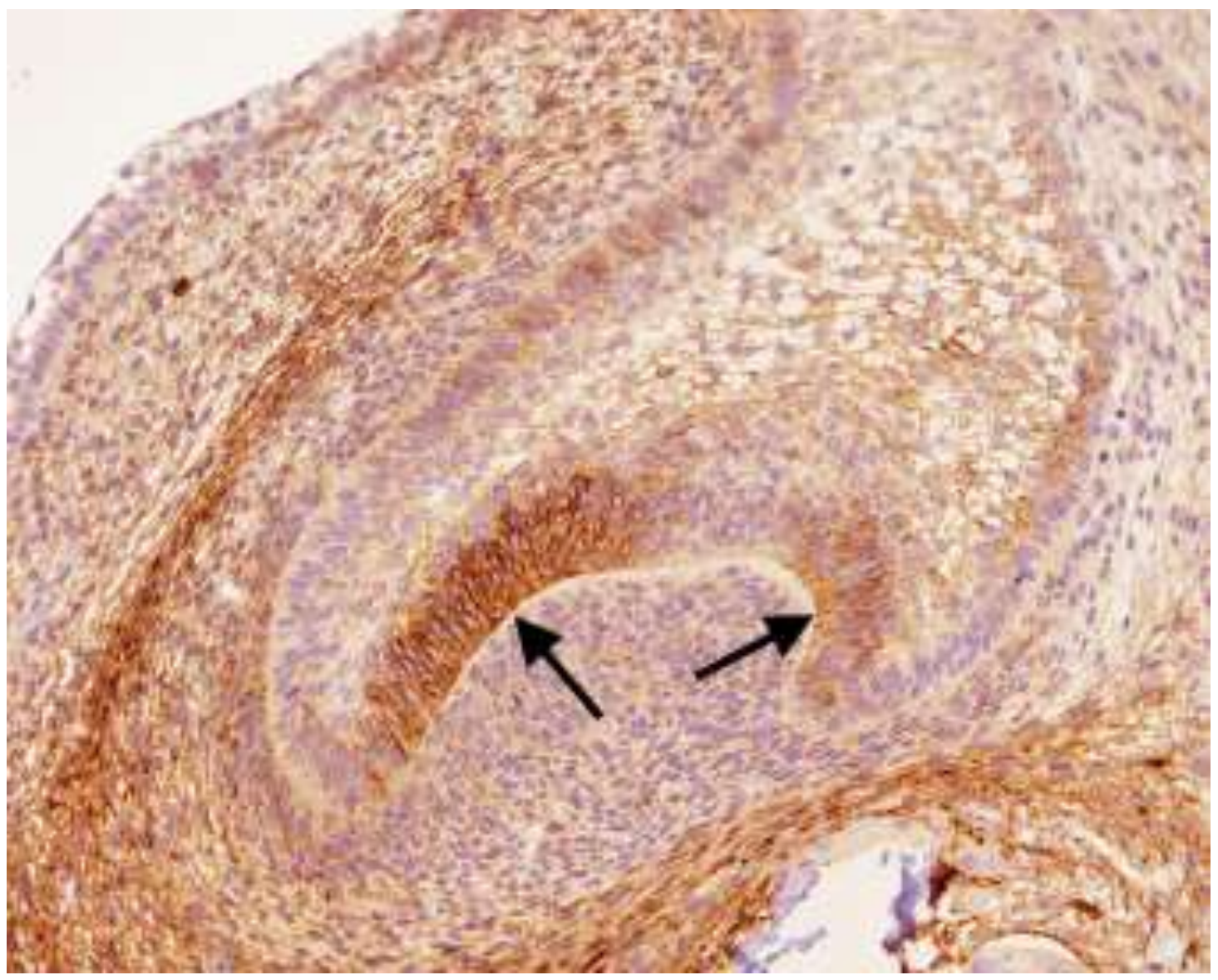

Fig. 2 - Positive staining of the OEE and SR with intense positivity of the IEE (arrow, excluding the central area associated with the enamel knot). Weak staining of the dental papilla with intense CD56 positivity in the dental follicle (original magnification X100). 


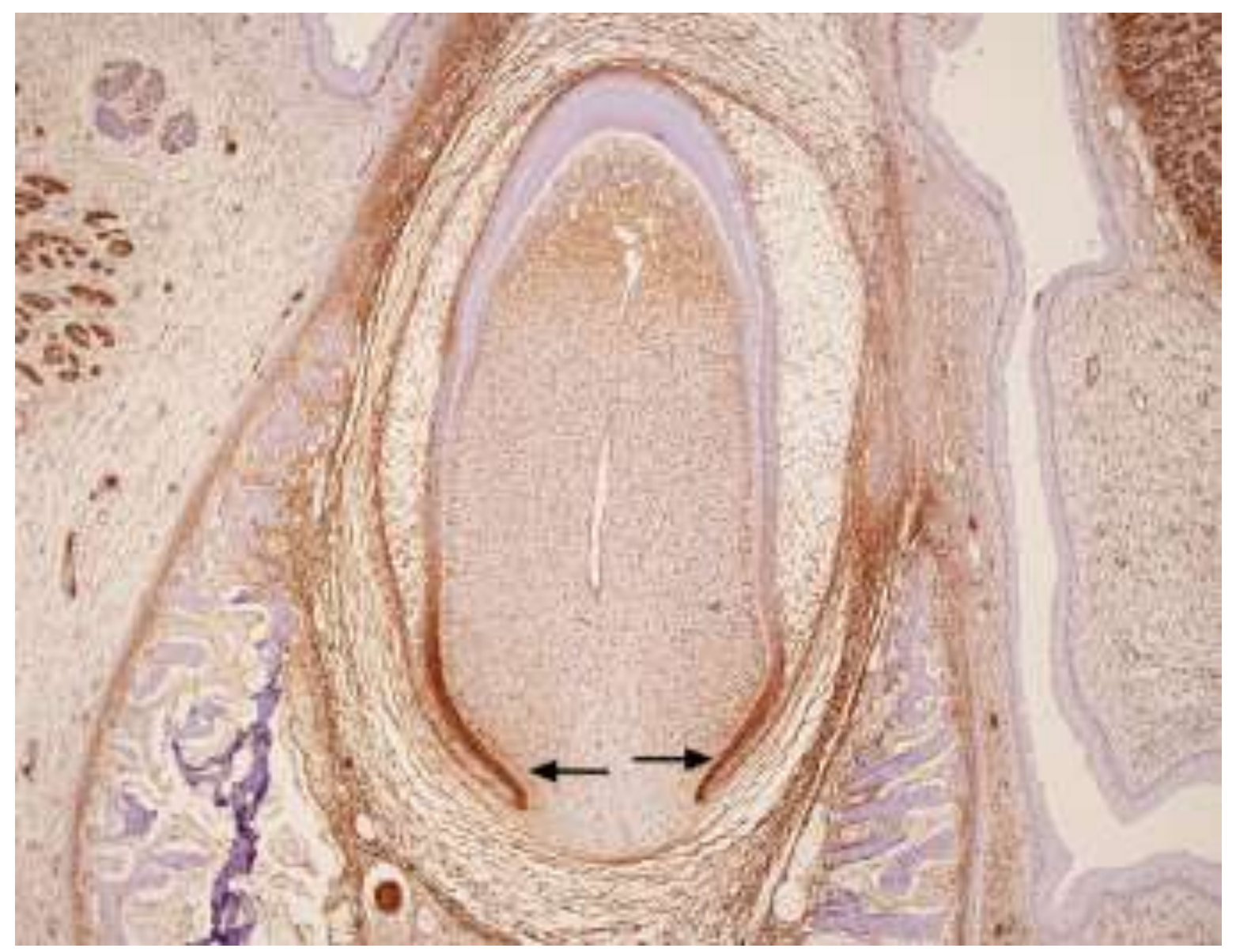

Fig. 3 - CD56 staining of the enamel organ in the bell stage. Intense positivity at the cervical loop areas including the IEE (arrows), SI, SR and OEE. The dental pulp and follicle also reveal positive staining for CD56. 


\section{Table 1 - CD56 staining patterns observed in the respective stages of tooth development.}

Developmental Location Weak Moderate Intense No

\begin{tabular}{|c|c|c|c|c|c|}
\hline stage & & staining & staining & staining & staining \\
\hline Bud & Odontogenic epithelium & $19 / 19$ & & & \\
\hline \multirow[t]{3}{*}{$n=19$} & Palisaded peripheral & & $13 / 19$ & $6 / 19$ & \\
\hline & odontogenic epithelial cells & & & & \\
\hline & Condensed ectomesenchyme & & & $19 / 19$ & \\
\hline Cap & OEE and SR & $5 / 12$ & $7 / 12$ & & \\
\hline \multirow[t]{4}{*}{$n=12$} & Central IEE & $12 / 12$ & & & \\
\hline & Peripheral IEE & & $5 / 12$ & $7 / 12$ & \\
\hline & Dental papilla & $12 / 12$ & & & \\
\hline & Dental follicle & & & $12 / 12$ & \\
\hline Bell & Differentiated ameloblasts & & & & $16 / 16$ \\
\hline \multirow[t]{6}{*}{$n=16$} & IEE, OEE and SR associated & & & $16 / 16$ & \\
\hline & with cervical loop & & & & \\
\hline & Dental papilla & & $16 / 16$ & & \\
\hline & Dental follicle & & & $16 / 16$ & \\
\hline & Dental lamina & & $11 / 14^{*}$ & $3 / 14^{*}$ & \\
\hline & Serres rests & & $11 / 14^{*}$ & $3 / 14 *$ & \\
\hline
\end{tabular}

* Dental laminae and Serres rests were identified in 14 of the bell stage sections. 


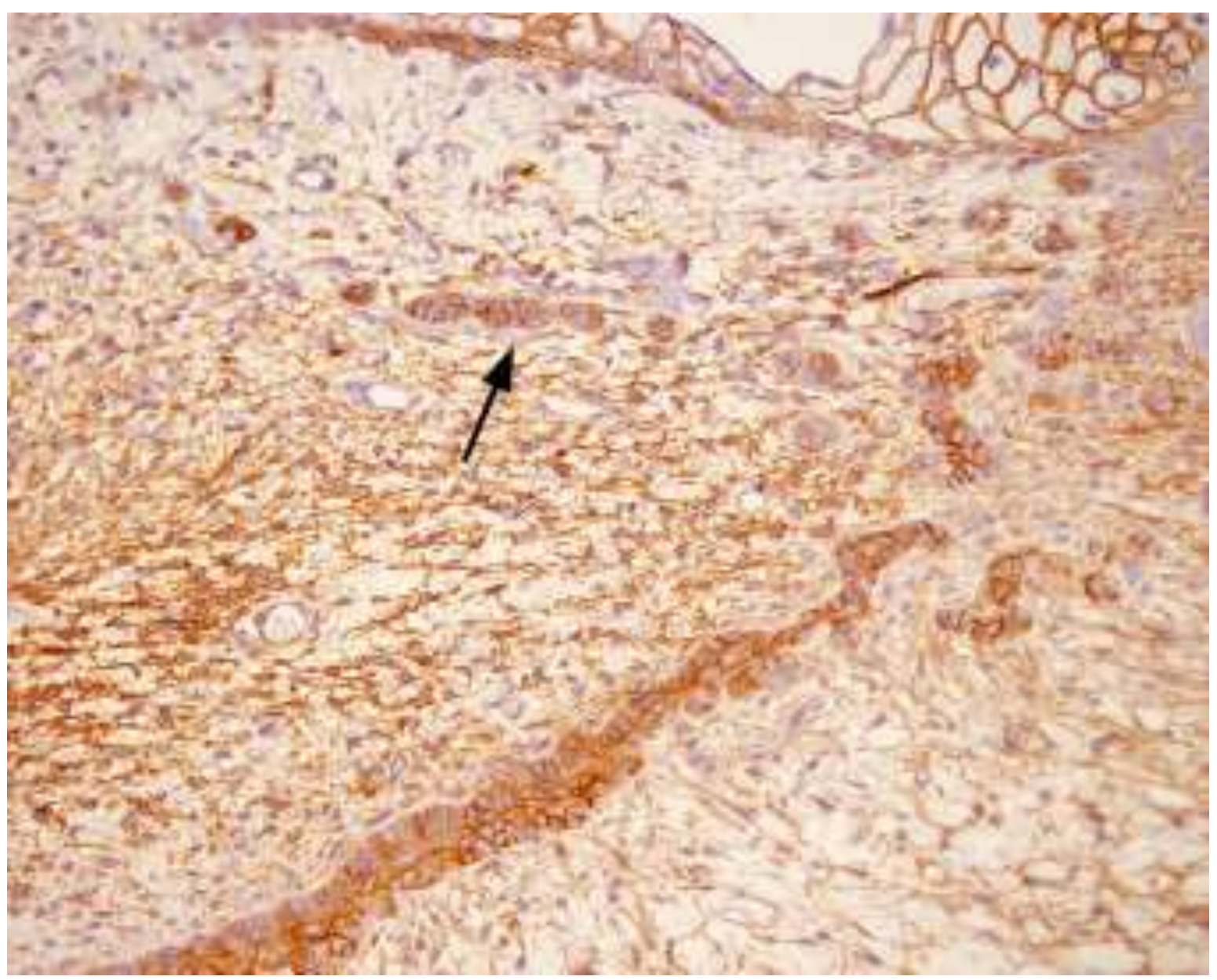

Fig. 4 - CD56 positive staining of the dental lamina and Serres rests (arrow). 
where the dental lamina and Serres rests were clearly identifiable, these structures constantly revealed CD56 positivity (Fig.4).

\section{Discussion}

The positive staining of the epithelial cells during the bud stage was more pronounced in the peripheral cells especially those in a more advanced stage of development. This is in contrasts with the findings of Obara et al. where the inner cells of the bud stage epithelium stained positive for CD56. ${ }^{15}$

The staining pattern observed in the cap stage is in agreement with the findings of Obara et al. as intense staining of the IEE adjacent to the enamel knot area corresponds to the "inner part of the enamel organ in a limited area close to the outer enamel epithelium". The positive staining of the papilla and follicle is also in concert with the staining patterns found in mice. ${ }^{15}$

Obara et al. described the enamel organ cells as mostly negative in the early and late bell stages with the exception of transient positivity in the SI. This is inconsistent with our findings as a constant pattern of intense positivity was observed in the cervical loop are including IEE, SI, SR and OEE. Although Obara et al. only detected transient staining in the cuspal aspect of the dental papilla at the early bell stage, the dental pulp revealed constant positivity associated with the cervical loop areas in all cases of the present study. The dental follicle however revealed strong positive staining throughout the experimental period in both studies. ${ }^{15}$ 
It has been speculated that CD56 expression pattern should be similar in human and murine tooth germs, ${ }^{12}$ but one should be careful in extrapolating results between species as mentioned earlier and supported by the results obtained in this study. The CD56 expression in mouse incisors have been shown to differ from that found in molar teeth, most probably as a result of the continuous eruption of mouse incisors compared to the limited eruption of molars. ${ }^{13}$ Another possible reason for the differences observed, could be the fact that CD56 is modulated by alternative RNA splicing, resulting in different cytoplasmic domains that differentially interact with the cell membrane. This regulatory mechanism may link CD56 binding function with other primary cell processes during embryonic development. ${ }^{1}$

CD56 distribution has been proven to exhibit dynamic changes during mouse molar development. ${ }^{16}$ Although CD56 is expressed in neural cells including Schwann cells, the pattern of expression thereof in the dental follicle differs from the pattern of innervation as revealed by a study correlating the anti-NF antibody expression. ${ }^{16}$ Loss of CD56 has been proposed to correlate with the differentiation of follicular cells. ${ }^{16}$ This is in agreement with the current results, as the IEE cells lost their CD56 expression as they differentiated into ameloblasts. Therefor CD56 may be involved in retaining the structure of the dental organ and regulate cell-cell interaction during odontogenesis. ${ }^{16}$

Various non-neuronal cells express CD56 during embryonic development and some of them (lung epithelium and dermal papilla of feathers) even express this molecule in adult life. ${ }^{20,21}$ It has been proposed that persistent CD56 expression in adult life reflects the retained potential of differentiation and morphogenesis even after development has ceased. ${ }^{16}$ Persistent expression of CD56 in the dental pulp have been proposed to correlate with the regenerative potential of the dental pulp as the CD56 expression may have 
marked undifferentiated ectomesenchymal cells. ${ }^{16}$ Another study on the dental follicle of impacted human third molars proposed that CD56 staining identified immature fibroblast cells with phenotypic features of stromal cells in the dental follicle and the authors agreed that CD56 may be used as a stem-cell marker as well as a marker for stromal phenotype. ${ }^{17}$

Even though the exact cells of origin of ameloblastomas is still unknown, ${ }^{22}$ the dental epithelial cells including the dental lamina and enamel organ cells seem to be responsible for these tumours. ${ }^{23}$ The remnants of the dental lamina, Serres rests, are therefor role players in the pathogenesis of ameloblastomas. In this study the dental lamina and Serres rests revealed positive staining for CD56 throughout the investigation. This correlates with the expression of CD56 in ameloblastomas. In $2001 \mathrm{Er}$ et al. described CD56 staining in the peripheral cells of follicular ameloblastoma tumor nests, with absence in the central stellate reticulum cells. ${ }^{11}$ This corresponds with the staining patterns observed in our study during the bud, cap and bell stage where certain cells stained intensely positive while others were devoid of CD56 staining. The proposal that the outer columnar cells of ameloblastomas may transdifferentiate to neuroectodermal cells should also be kept in mind. ${ }^{12}$

In conclusion, CD56 expression in odontogenesis is spatially and temporally regulated resulting in heterogeneous expression patterns during the different stages of tooth development. It is positively expressed in the dental lamina and Serres rests which correlate with the expression thereof found in ameloblastomas. Keeping the physiological functions of cell adhesion molecules in mind, CD56 might play a crucial role in tumour biology. It may be implicated in alterations in the cell membrane environment which in turn could increase the local aggressive growth potential of ameloblastomas. However the distinct manner remains to be resolved. 


\section{Acknowledgments}

We would like to thank the Veterinary Academic Hospital at Onderstepoort, Dr G

Steenkamp and Dr. MF Visser (Lakeside Veterinary Clinic) for providing us with the canine foetuses used in this study. 


\section{REFERENCES}

1. Cunningham BA, Hemperly JJ, Murray BA, Prediger EA, Brackenbury R, Edelman GM. Neural cell adhesion molecule: structure, immunoglobulin-like domains, cell surface modulation, and alternative RNA splicing. Science 1987;236(4803):799-806.

2. Lanier LL, Chang C, Azuma M, Ruitenberg JJ, Hemperly JJ, Phillips JH. Molecular and functional analysis of human natural killer cell-associated neural cell adhesion molecule ( $\mathrm{N}$ CAM/CD56). J Immunol 1991;146(12):4421-4426.

3. Le Forestier N, Lescs MC, Gherardi RK. Anti-NKH-1 antibody specifically stains unmyelinated fibres and non-myelinating Schwann cell columns in humans. Neuropathol Appl Neurobiol 1993;19(6):500-506.

4. Peterson PE, Blankenship TN, Wilson DB, Hendrickx AG. Analysis of hindbrain neural crest migration in the long-tailed monkey (Macaca fascicularis). Anat Embryol (Berl) 1996;194(3):235-246.

5. Zeromski J, Nyczak E, Dyszkiewicz W. Significance of cell adhesion molecules, CD56/NCAM in particular, in human tumor growth and spreading. Folia Histochem Cytobiol 2001;39 Suppl 2:36-37.

6. Sinanan AC, Hunt NP, Lewis MP. Human adult craniofacial muscle-derived cells: neural-cell adhesion-molecule (NCAM; CD56)-expressing cells appear to contain multipotential stem cells. Biotechnol Appl Biochem 2004;40(Pt 1):25-34.

7. Kraj M, Sokolowska U, Kopec-Szlezak J, Poglod R, Kruk B, Wozniak J, et al. Clinicopathological correlates of plasma cell CD56 (NCAM) expression in multiple myeloma. Leuk Lymphoma 2008;49(2):298-305. 
8. Cho EY, Choi Y, Chae SW, Sohn JH, Ahn GH. Immunohistochemical study of the expression of adhesion molecules in ovarian serous neoplasms. Pathol Int 2006;56(2):62-70.

9. Farinola MA, Weir EG, Ali SZ. CD56 expression of neuroendocrine neoplasms on immunophenotyping by flow cytometry: a novel diagnostic approach to fine-needle aspiration biopsy. Cancer 2003;99(4):240-246.

10. Cairns L, Naidu A, Robinson CM, Sloan P, Wright JM, Hunter KD. CD56 (NCAM) expression in ameloblastomas and other odontogenic lesions. Histopathology 2010;57(4):544-548.

11. Er N, Dagdeviren A, Tasman F, Zeybek D. Neural cell adhesion molecule and neurothelin expression in human ameloblastoma. J Oral Maxillofac Surg 2001;59(8):900903; discussion 904.

12. Kusafuka K, Hirobe K, Wato M, Tanaka A, Nakajima T. CD56 expression is associated with neuroectodermal differentiation in ameloblastomas: an immunohistochemical evaluation in comparison with odontogenic cystic lesions. Med Mol Morphol 2011;44(2):7985.

13. Obara N, Takeda M. Distribution of the neural cell adhesion molecule (NCAM) during pre- and postnatal development of mouse incisors. Anat Embryol (Berl) 1997;195(2):193202.

14. Obara N. Expression of the neural cell adhesion molecule during mouse tooth development. Connect Tissue Res 2002;43(2-3):212-215.

15. Obara N, Suzuki Y, Nagai Y, Nishiyama H, Mizoguchi I, Takeda M. Expression of neural cell-adhesion molecule mRNA during mouse molar tooth development. Arch Oral Biol 2002;47(11):805-813. 
16. Obara N, Takeda M. Expression of neural cell adhesion molecule (NCAM) during the first molar development in the mouse. Anat Embryol (Berl) 1993;187(3):209-219.

17. Angiero F, Rossi C, Ferri A, Seramondi R, Magistro S, Farronato D, et al. Stromal phenotype of dental follicle stem cells. Front Biosci (Elite Ed) 2012;4:1009-1014.

18. Degistirici O, Jaquiery C, Schonebeck B, Siemonsmeier J, Gotz W, Martin I, et al. Defining properties of neural crest-derived progenitor cells from the apex of human developing tooth. Tissue Eng Part A 2008;14(2):317-330.

19. Lewis JR, Reiter AM, Mauldin EA, Casal ML. Dental abnormalities associated with Xlinked hypohidrotic ectodermal dysplasia in dogs. Orthod Craniofac Res 2010;13(1):40-47. 20. Chuong CM, Edelman GM. Expression of cell-adhesion molecules in embryonic induction. II. Morphogenesis of adult feathers. J Cell Biol 1985;101(3):1027-1043.

21. Crossin KL, Chuong CM, Edelman GM. Expression sequences of cell adhesion molecules. Proc Natl Acad Sci U S A 1985;82(20):6942-6946.

22. Heikinheimo K, Kurppa KJ, Laiho A, Peltonen S, Berdal A, Bouattour A, et al. Early Dental Epithelial Transcription Factors Distinguish Ameloblastoma from Keratocystic Odontogenic Tumor. J Dent Res 2014.

23. Heikinheimo K, Jee KJ, Niini T, Aalto Y, Happonen RP, Leivo I, et al. Gene expression profiling of ameloblastoma and human tooth germ by means of a cDNA microarray. J Dent Res 2002;81(8):525-530. 